\title{
MUSCLE COORDINATION OF SUPPORT, PROGRESSION AND BALANCE DURING STAIR AMBULATION
}

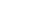

Yi-Chung Lin, Laurence A. Fok, Anthony G. Schache and Marcus G. Pandy

Dept of Mechanical Engineering, University of Melbourne, Parkville, Victoria,

Australia

Word count: 3,949 (Introduction to Discussion)

4 Address correspondence to:

Yi-Chung Lin, Ph.D.

Department of Mechanical Engineering

University of Melbourne

Parkville, Victoria 3010, Australia

Ph. +61 383440405

Fax. +61383444290

Email: $\underline{\text { linyc@unimelb.edu.au }}$ 
2 Stair ambulation is more physically demanding than level walking because it requires

3 the lower-limb muscles to generate greater net joint moments. Although lower-limb

4 joint kinematics and kinetics during stair ambulation have been extensively studied,

5 relatively little is known about how the lower-limb muscles accelerate the whole-body

6 center of mass (COM) during stair ascent and descent. The aim of the current study

7 was to evaluate differences in muscle contributions to COM accelerations between

8 level walking and stair ambulation in 15 healthy adults. Three-dimensional

9 quantitative gait analysis and musculoskeletal modeling were used to calculate the

10 contributions of the individual lower-limb muscles to the vertical, fore-aft and

11 mediolateral accelerations of the COM (support, progression, and balance, respectively) during level walking, stair ascent and stair descent. Muscles that contribute most significantly to the acceleration of the COM during level walking (hip, knee, and ankle extensors) also dominate during stair ambulation, but with noticeable differences in coordination. In stair ascent, gluteus maximus accelerates the body forward during the first half of stance and soleus accelerates the body backward during the second half of stance, opposite to the functions displayed by these muscles Keywords: gait, falls prevention, induced accelerations, muscle function 
Stair ambulation is an activity of daily living. Although healthy adults can perform this task with relative ease, ascending and descending stairs can be more demanding for people with compromised motor function, such as the elderly (Reeves et al., 2008) or individuals with osteoarthritis (Kaufman et al., 2001; Asay et al., 2009). Compared to level walking, stair ambulation is associated with greater risk of severe or fatal falls (Manning, 1983), where $75 \%$ of these falls occur during stair descent (Svanström, 1974; Tinetti et al., 1988). Since muscles are responsible for controlling body movement, a better understanding of how muscles accelerate the whole-body center of mass (COM) (henceforth referred to as muscle function) during stair ambulation could help facilitate the development of more effective fall prevention strategies.

Lower-limb muscle function during level walking has been extensively investigated using musculoskeletal modelling approaches. Each muscle contributes to the vertical, fore-aft and mediolateral accelerations of the COM during stance (described as support, progression, and balance, respectively) (Pandy and Andriacchi, 2010). Liu et al. (2006) and Pandy et al. (2010) reported that gluteus medius, gluteus maximus, vasti, and soleus contribute significantly to support in the first half of stance, whereas forward progression in the second half of stance is dominated by soleus and gastrocnemius. Furthermore, to maintain balance in the frontal plane, Pandy et al. (2010) and John et al (2012) showed that gluteus medius coordinates with vasti in the first half of stance while gluteus medius coordinates with both soleus and gastrocnemius in the second half of stance. By comparison, less is known about how the lower-limb muscles coordinate motion of the COM during stair ambulation. 
1 Inverse dynamics-based studies suggest that greater knee and ankle extension moments are exerted during the first half of stance in stair ambulation than during this period in level walking (Riener et al., 2002; Silverman et al., 2014). These studies have also shown the peak knee extension moment during the second half of stance in stair descent to be more than three-fold greater than that observed during level walking. Finally, the ankle plantarflexion moment can peak as high as $75 \%$ of a maximal voluntary contraction in the early stance phase of stair descent (Reeves et al., 2008), whereas the ankle plantarflexion moment peaks during late stance in level walking.

Inverse dynamics-based studies have also investigated differences in the hip joint moment between level walking and stair ambulation. Compared to level walking, Riener et al. (2002) and Silverman et al. (2014) found the peak hip extension moment in the early stance phase of stair ascent and descent to be significantly smaller, with a larger reduction evident during stair descent. A few studies have compared the peak hip abduction moment between level walking and stair ambulation and have reported some inconsistent results. For example, Silverman et al. (2014) found the first and second peaks of the hip abduction moment during stair ascent to be significantly lower than those measured for level walking, whereas Nadeau et al. (2003) found no significant difference in the magnitude of the first peak.

While the aforementioned studies have provided important insights into the differences in net joint moments between level walking and stair ambulation, the corresponding changes in the functional roles of the individual lower-limb muscles can only be inferred from these differences (Zajac and Gordon, 1989). The reported differences in the magnitudes and/or timing of the lower-limb joint moments between level walking and stair ambulation suggest that there may also be differences between 
these two activities in the way the hip, knee, and ankle extensor muscles coordinate motion of the COM.

In the present study, we used a three-dimensional musculoskeletal model to investigate how lower-limb muscle function during stair ambulation differs from that during level walking. We anticipated that any differences in muscle contributions to COM motion will most likely be evident in the vertical direction because of the roles of the hip, knee, and ankle extension moments in supporting the body (Kepple et al., 1997) and the need to control the vertical COM displacement during stair ambulation. Given that stair ambulation has been demonstrated to be associated with greater knee and ankle extension moments but a reduced hip extension moment compared to level walking, the vertical support provided by these three extension moments should also vary accordingly. We therefore hypothesized that during stair ambulation the peak contributions to the vertical acceleration of the COM would be significantly increased for the knee and ankle extensors but significantly reduced for the hip extensors. The results of this study will provide insight into which lower-limb muscles are most relied upon for support, progression and balance during stair ambulation, and thus likely play a pivotal role in preventing stair-related falls. height: $166 \pm 8 \mathrm{~cm}$ ) underwent gait experiments in the Biomotion Laboratory at the Fifteen healthy adults ( 4 males, 11 females; age: $54 \pm 8$ yrs; weight: $67 \pm 11 \mathrm{~kg}$; University of Melbourne. Ethical approval was obtained from the University of Melbourne Human Research Ethics Committee, and each participant provided written informed consent prior to the commencement of the study. Reflective markers were placed at specific anatomical landmarks on the trunk, pelvis, and both arms and legs. 
1 Marker trajectories were captured at $120 \mathrm{~Hz}$ using a nine-camera motion capture system (Vicon, Oxford Metrics Ltd, Oxford, UK) during all locomotor tasks. Pairs of $\mathrm{Ag} / \mathrm{AgCl}$ surface electrodes (MediMax Global, Shalden, Hampshire, UK) were placed on an arbitrarily chosen leg to record the electromyographic (EMG) signal from five muscles: gluteus maximus, gluteus medius, vastus lateralis, gastrocnemius, and soleus. Additional details regarding retro-reflective marker and EMG electrode placement have been reported previously (Crossley et al., 2012). Ground reaction forces (GRFs) during gait were measured using a series of three ground-embedded force plates (Advanced Mechanical Technology Inc., Watertown, MA, USA), whereas GRFs during stair ambulation were measured using one ground-embedded force plate and two portable AccuGait force plates (Advanced Mechanical Technology Inc., Watertown, MA, USA) mounted on the first and second steps of a custom-built three-step staircase. GRF and EMG data were sampled at $1080 \mathrm{~Hz}$.

All participants performed level walking $(1.36 \pm 0.15 \mathrm{~m} / \mathrm{s})$, stair ascent $(0.50 \pm$ $0.11 \mathrm{~m} / \mathrm{s})$ and stair descent $(0.74 \pm 0.20 \mathrm{~m} / \mathrm{s})$ tasks at a self-selected speed while wearing standardized footwear. Participants were asked to stand still in their neutral pose before performing any task. They were then instructed to land their test leg on the second ground-embedded force plate and the first step of the staircase during level walking and stair ambulation, respectively. Each trial commenced from initial contact with the test leg, and only data for the stance phase were analyzed. EMG data were also collected whilst all participants performed isometric maximum voluntary contractions of the muscles crossing the hip, knee and ankle. Marker and GRF data were low-pass filtered at 4 and $60 \mathrm{~Hz}$, respectively, using a fourth-order Butterworth filter. EMG data were full-wave rectified and low-pass filtered at $10 \mathrm{~Hz}$ using a second-order Butterworth filter to create linear envelopes, which were normalized by 
the mean EMG signals recorded from each subject's maximum voluntary contraction trials.

A generic three-dimensional musculoskeletal model was implemented in an opensource software package (Delp et al., 2007) to calculate joint kinematics, joint kinetics and muscle forces based on the experimental data. The skeleton was represented as a 12-segment, 23 degree-of-freedom linkage system. The head and trunk were modelled as a single rigid body that articulated with the pelvis via a ball-and-socket joint. For the lower limbs, each hip was modelled as a ball-and-socket joint, each knee as a translating hinge joint, and each ankle as a universal joint comprised of two nonintersecting hinge joints. The lower limbs and trunk were actuated by 92 muscletendon units, with each unit represented as a three-element Hill-type muscle in series with an elastic tendon (Zajac, 1989). For the upper limbs, each shoulder was modelled as a ball-and-socket joint and each elbow was represented as a universal joint comprised of two non-intersecting hinge joints. The joints of the upper limbs were actuated by ten ideal torque motors (Dorn et al., 2012).

Scaled-generic models were developed by scaling the segmental inertial properties and muscle-tendon attachment sites assumed in the generic musculoskeletal model to each participant's body dimensions. Joint angles were computed over an entire gait cycle using an inverse kinematics analysis that minimized the sum of the squared differences between the positions of virtual markers identified on the model and reflective markers placed on the subject ( $\mathrm{Lu}$ and O'Connor, 1999). Internal joint moments were calculated using a standard inverse dynamics approach.

Joint moments were decomposed into individual muscle forces using a static optimization algorithm, which minimized the sum of all muscle activations squared subject to each muscle's force-length-velocity properties (Anderson and Pandy, 
1 2001). A pseudo-inverse force decomposition method (Lin et al., 2011) was then used to compute the contributions of all lower-limb muscle forces to the vertical, fore-aft, and mediolateral accelerations of the COM (support, progression, and balance, respectively). Individual muscle forces, as well as their contributions to the COM accelerations, were combined into functional muscle groups (see Figure 2 caption). All results were time-normalized to the stance phase and then averaged separately across all participants. Muscle forces and joint moments were normalized to each participant's body weight and to body weight and height, respectively.

One-way repeated-measures ANOVA tests were used to determine whether locomotor task (i.e., level walking, stair ascent, and stair descent) significantly influenced the peak muscle forces and peak muscle contributions to the COM accelerations. If a significant main effect was obtained, post hoc paired $t$-tests were used to determine if significant differences existed between each of the locomotor tasks. A significance level of $p<0.017$ was set for all tests after applying a Bonferroni correction to the significance level of 0.05 (i.e., three pairwise comparisons were performed per dependent variable). Note that only the pairwise comparisons of stair ambulation versus level walking were of interest; the pairwise comparison of stair ascent versus descent was beyond the scope of the present study.

\footnotetext{
RESULTS

In the sagittal plane, stair ascent and descent both required greater peak moments at the knee and ankle joints in the first half of the stance phase, but a smaller peak moment at the ankle joint in the second half of stance when compared to level walking (Fig. 1). During stair ascent a hip extension moment was present throughout the stance phase. The peak hip extension and flexion moments were reduced during
} 
the first and second half of stance, respectively, in stair descent relative to level walking. In the frontal plane, a double-bump hip abduction moment was observed across all three functional tasks, but the magnitude of this moment was reduced during stair ascent.

The time histories of the predicted muscle forces were in general agreement with the recorded EMG linear envelopes for level walking and stair ambulation, except for SOL during stair descent and GMED during stair ascent (Fig. 2). Locomotor task had a significant effect on peak muscle forces (Table 1). Post hoc tests revealed that differences in peak muscle forces were evident during both stair ascent and stair descent compared to level walking (Fig. 3). During stair ascent, the peak forces generated by VAS and SOL in the first half of stance were significantly higher than during level walking $(p<0.001)$, whereas the peak forces generated by SOL, GMED, and GAS in the second half of stance were significantly lower than during level walking (SOL: $p=0.003$; GMED and GAS: $p<0.001$ ). Over the entire stance phase for stair descent, the force generated by SOL was higher than that generated during level walking, with the magnitude of the peak force being significantly different $(p=$ 0.003). The peak force generated by VAS in the second half of the stance phase for stair descent was significantly higher $(p<0.001)$ than that generated during level walking, whereas the peak force generated by GAS was significantly lower $(p<$ $0.001)$.

Locomotor task had a significant effect on muscle function during the first half of stance for the hip, knee, and ankle extensors and the hip abductors; the fore-aft contributions of VAS and SOL, however, were not significantly different (Table 1). Post hoc tests revealed that during stair descent GMAX and GMED generated significantly less vertical support compared to level walking (GMAX: $p<0.001$; 
1 GMED: $p<0.001$ ), whereas SOL generated significantly greater vertical support $(p<$ $20.001)$ and lateral acceleration $(p<0.001)$ (Fig. 5). The contributions of GMAX and

3 GMED to the fore-aft acceleration of the COM were also significantly different

4 between level walking and stair ambulation (GMAX: $p<0.001$; GMED: $p<0.015$ ).

5 Both hip muscles accelerated the body forward during the first half of stance in stair ambulation, whereas the same muscles decelerated the body during level walking (Figs. 4 and 5). GMED also provided significantly higher medial acceleration during stair descent $(p<0.001)$.

Locomotor task also had a significant effect on muscle function during the second half of stance for the hip, knee, and ankle extensors (Table 1). Post hoc tests revealed that VAS generated significantly greater vertical support $(p<0.002)$ and fore-aft deceleration $(p<0.003)$ during stair ambulation, whereas this muscle contributed virtually nothing during level walking (Figs. 4 and 6). Compared to level walking, VAS also generated significantly greater medial acceleration during stair descent $(p<$ 0.001). Contributions from SOL to accelerate the COM forward were not significantly different between level walking and stair descent; in contrast, SOL applied a backward acceleration during stair ascent. muscles to the generation of vertical support, the modulation of forward progression, and the control of mediolateral balance during stair ambulation in healthy adults. Consistent with our hypothesis, vertical support generated by the knee and ankle extensors was significantly greater during stair ascent and descent, respectively, than during level walking. Vertical support generated by the hip extensors was 
1 significantly less during stair descent, which also concurs with our hypothesis. Interestingly, the contributions of the hip extensors to the fore-aft acceleration of the COM differed in direction between level walking and stair ambulation.

Muscles that contribute most significantly to the acceleration of the COM during level walking (i.e., the hip, knee, and ankle extensors) also dominate the acceleration of the COM during stair ambulation, but with noticeable differences in coordination. For example, SOL generated the greatest forward acceleration during the second half of stance in level walking, while it generated the greatest backward acceleration during the same period of stair ascent. These differences can be attributed to the configuration of the skeletal system by calculating a given muscle's potential contribution to the acceleration of the COM on a unit-force basis. Such a calculation describes the net effect of body posture, and hence musculoskeletal geometry (i.e., moment arm) on the functional capacity of the muscle during a prescribed task (Correa and Pandy, 2013) (see Supplementary Material).

In agreement with Riener et al. (2002) and Silverman et al. (2014), we found that stair ascent and descent required greater knee and ankle extension moments during the first half of stance compared to level walking (Fig. 1). These increases in the extension moments were reflected in the greater forces generated by VAS and SOL during stair ambulation (Figs. 2 and 3), which is consistent with findings reported by Ghafari et al. (2009). The observed increase in the vertical support generated by these two extensors during stair ambulation supports our hypothesis.

Stair ascent is a more challenging functional task than level walking as the lowerlimb muscles must raise the COM against gravity in order to progress to the next step. Consistent with McFadyen and Winter (1988), we found that the knee extensors, VAS, played a primary role in supporting the body during the first half of stance (Fig. 
1 4). The hip and ankle extensors, GMAX and SOL, were the other two important muscles that contributed to the elevation of the COM during the same period of stair ascent.

The function of the largest muscle in the human body, GMAX, is likely to be of clinical relevance in terms of preventing backward falls during stair ascent. GMAX supported the body during both level walking and stair ascent. It also contributed greatly to the forward acceleration of the COM during stair ascent and the backward acceleration of the COM during level walking. This contribution to the body's forward movement during stair ascent may be the main mechanism that moves the COM closer to the center of pressure (which was described by McFadyen and Winter (1988) as an optimal position) during the first double support period in stair ascent. People with GMAX weakness may therefore experience difficulty in initiating stair ascent due to an inability to move the body's COM forward to this optimal position. Moreover, Zachazewski et al. (1993) has shown that the COM is anterior to the center of pressure for most of the single-leg support period during stair ascent. This relative anterior position of the COM probably helps to reduce the likelihood of a backward fall during stair ascent and it may be partially attributable to trunk flexion (Krebs et al., 1992), which likely requires optimal hip extensor function. Weak hip extensors are known to cause backward trunk lean during level walking (Perry, 1992); therefore, people with GMAX dysfunction will likely experience difficulty in controlling locomotor stability during stair ascent.

Consistent with the findings of McFadyen and Winter (1988), the ankle extensors, SOL and GAS, dominated vertical support during the second half of stance in stair ascent (Fig. 4). McFadyen and Winter (1988) also reported that the ankle extensors were involved in the forward movement of the body during the same period of stair 
1 ascent. We found that whilst SOL and GAS both contributed substantially to vertical support, GAS accelerated the COM forward whereas SOL produced a backward acceleration (Fig. 4). The action of GAS as a knee flexor may explain its contribution to forward acceleration during stair ascent, because its action as an ankle extensor would presumably decelerate the body as suggested by the contribution of SOL to backward acceleration.

During stair descent, humans are required to control the rate of lowering of the COM while progressing to the next step. Our results showed that VAS and SOL were the two major extensors to prevent the body from collapsing under the force of gravity and to control its speed during stair descent (Fig. 4), which is in agreement with the findings of McFadyen and Winter (1988).

The present study highlighted two interesting features of muscle coordination related to the knee extensors and hip abductors during stair descent. First, the contributions of VAS and GMED to the fore-aft acceleration of the COM during stance were similar in magnitude but opposite in direction; VAS and GMED decelerated and accelerated the body, respectively (Fig. 4). GMED is known for its role in providing frontal-plane stability during level walking (Winter 1995; Pandy et al., 2010; John et al., 2012), but our results showed that GMED also has a major role in propelling the body forward during stair descent. This functional role of GMED is especially important during the first half of stance as most of the forward acceleration of the COM is generated by GMED. For the same reason, the decelerating ability of VAS is especially critical during the midstance phase of stair descent. Therefore, the body may experience excessive forward momentum if VAS fails to provide the necessary fore-aft deceleration during stair descent, which would threaten locomotor stability and may induce a fall. 
during the second half of the stance phase in stair descent (Fig. 4). VAS has been reported to accelerate the body laterally during early stance in level walking (Pandy et al., 2010; John et al., 2012). In the current study, VAS also accelerated the body laterally during early stance in both level walking and stair ascent; however, it accelerated the body medially during the transition from single-leg support to doubleleg support in stair descent (Fig. 4). Therefore, VAS and GMED acted in unison to control frontal-plane balance by maintaining the projection of the COM medial to the base of support during this transition period. The quadriceps muscles play an important role in stair descent, and quadriceps strength is known to decrease significantly with increasing age (Hurley et al., 1988). It is possible that this reduction in quadriceps strength impedes the ability of the quadriceps to maintain frontal-plane balance and may explain why older adults fall more frequently during stair descent than ascent (Svanström, 1974; Tinetti et al., 1988).

Three limitations should be considered when interpreting the results of this study. First, muscle forces were calculated by solving a static optimization problem that minimized the sum of the muscle activations squared. Although this performance criterion has been widely used to predict muscle forces during level walking (Glitsch and Baumann, 1997; Pandy and Andriacchi, 2010; Lin et al., 2012), it is unclear whether the same criterion is also applicable to stair ambulation. Nevertheless, the temporal agreement between our muscle force predictions and the measured EMG linear envelopes was generally good for level walking and stair ambulation (Fig. 2). We note, however, that the calculated SOL force during stair descent is greater than may be expected based on the recorded EMG activity, whereas the calculated GMED force during stair ascent is smaller than expected. We examined the potential causes 
1 of these two discrepancies and concluded that they are unlikely to significantly affect our interpretations of SOL and GMED function (see Supplementary Material).

Second, no published results are available for comparison with our results of lower-limb muscle function during stair ambulation. However, our muscle function results for level walking are consistent with those published previously (Neptune et al., 2004; Liu et al., 2006; Pandy et al., 2010; John et al., 2012). Since the same musculoskeletal model and methods were used to calculate muscle function during level walking and stair ambulation for each subject, we have confidence in the conclusions derived for stair ambulation. In addition, our GRF measurements (Fig. S2, Supplementary Material) and calculated joint kinematics (Figs. S3 and S4, Supplementary Material) during stair ambulation are consistent with results reported previously by others (Nadeau et al., 2003; Protopapadaki et al., 2007; Reeves et al., 2008; Bovi et al., 2011).

Third, a three-step staircase was used in the present study, which limits the ability to analyze steady-state stair ambulation. While knee kinematics has been found to remain consistent across gait cycles during stair ambulation on a four-step staircase (Whatling and Holt, 2010), Cluff and Robertson (2011) demonstrated that a single gait cycle may not be sufficient to achieve steady-state stair descent. Further work is required to understand the extent to which the number of stair steps affects muscle function during stair ambulation.

To summarize, we observed several distinct differences in the coordination of the major lower-limb muscles between level walking and stair ambulation. In stair ascent, GMAX accelerates the body forward and SOL accelerates the body backward during the first and second half of stance, respectively, opposite to the functions displayed by these muscles in level walking. In stair descent, VAS generates 
1 backward and medial accelerations of the COM during the second half of stance,

2 whereas it contributes minimally during this period in level walking. Previous studies

3 have shown that muscle strength training can reduce the risk of falling (Horlings et

4 al., 2008; Seguin and Nelson, 2003). Our findings related to the functional roles of

5 hip, knee, and ankle extensors during stair ambulation further suggest that optimizing

6 the performance of these muscles should be prioritized in programs aimed at 7 preventing stair-related falls.

8

\section{ACKNOWLEDGMENTS}

This work was supported by an Australian Research Council Grant (DP0878705), a

National Health and Medical Research Council of Australia Project Grant (508966), and a VESKI Innovation Fellowship awarded to MGP. We thank Jonathan Lentzos and Hannah Ozturk for their assistance with the collection of gait data.

4

\section{CONFLICT OF INTEREST STATEMENT}

None of the authors have a conflict of interest in relation to the work reported here. 
Anderson, F.C., Pandy, M.G., 2001. Static and dynamic optimization solutions for gait are practically equivalent. Journal of Biomechanics 34, 153-161.

Asay, J.L., Mündermann, A., Andriacchi, T.P., 2009. Adaptive patterns of movement during stair climbing in patients with knee osteoarthritis. Journal of Orthopaedic Research 27, 325-329.

Bovi, G., Rabuffetti, M., Mazzoleni, P., \& Ferrarin, M., 2011. A multiple-task gait analysis approach: kinematic, kinetic and EMG reference data for healthy young and adult subjects. Gait Posture, 33, 6-13.

Cluff, T., Robertson, D. G., 2011. Kinetic analysis of stair descent: Part 1. Forwards step-over-step descent. Gait Posture, 33, 423-428.

Crossley, K.M., Dorn, T.W., Ozturk, H., Van den Noort, J., Schache, A.G., Pandy, M.G., 2012. Altered hip muscle forces during gait in people with patellofemoral osteoarthritis. Osteoarthritis and Cartilage 20, 1243-1249.

Delp, S.L., Anderson, F.C., Arnold, A.S., Loan, P., Habib, A., John, C.T., Guendelman, E., Thelen, D.G., 2007. OpenSim: Open-source software to create and analyze dynamic simulations of movement. IEEE Transactions on Biomedical Engineering 54, 1940-1950.

Dorn, T.W., Schache, A.G., Pandy, M.G., 2012. Muscular strategy shift in human running: dependence of running speed on hip and ankle muscle performance. $\mathrm{J}$ Exp Biol, 215, 1944-1956.

Dwyer, M. K., Stafford, K., Mattacola, C. G., Uhl, T. L., Giordani, M., 2013. Comparison of gluteus medius muscle activity during functional tasks in individuals with and without osteoarthritis of the hip joint. Clinical Biomechanics, 28, 757-761.

Fok, L.A., Schache, A.G., Crossley, K.M., Lin, Y.-C., Pandy, M.G., 2013. Patellofemoral joint loading during stair ambulation in people with patellofemoral osteoarthritis. Arthritis and Rheumatism 65, 2059-2069.

Foucher, K. C., Hurwitz, D. E., \& Wimmer, M. A., 2007. Preoperative gait adaptations persist one year after surgery in clinically well-functioning total hip replacement patients. Journal of Biomechanics, 40, 3432-3437.

Ghafari, A.S., Meghdari, A., Vossoughi, G.R., 2009. Muscle-driven forward dynamics simulation for the study of differences in muscle function during stair ascent and descent. Proceedings of the Institution of Mechanical Engineers, Part H: Journal of Engineering in Medicine 223, 863-874.

Glitsch, U., Baumann, W., 1997. The three-dimensional determination of internal loads in the lower extremity. Journal of Biomechanics 30, 1123-1131.

Hicks-Little, C.A., Peindl, R.D., Fehring, T.K., Odum, S.M., Hubbard, T.J., Cordova, M.L., 2012. Temporal-spatial gait adaptations during stair ascent and descent in patients with knee osteoarthritis. Journal of Arthroplasty 27, 1183-1189.

Horlings, C.G.C., van Engelen, B.G.M., Allum, J.H.J., Bloem, B.R., 2008. A weak balance: the contribution of muscle weakness to postural instability and falls. Nat Clin Pract Neuro 4, 504-515.

Hurley, M.V., Rees, J., Newham, D.J., 1998. Quadriceps function, proprioceptive acuity and functional performance in healthy young, middle-aged and elderly subjects. Age Ageing, 27, 55-62. 
John, C.T., Seth, A., Schwartz, M.H., Delp, S.L., 2012. Contributions of muscles to mediolateral ground reaction force over a range of walking speeds. Journal of Biomechanics, 45, 2438-2443.

Kaufman, K.R., Hughes, C., Morrey, B.F., Morrey, M., An, K.-N., 2001. Gait characteristics of patients with knee osteoarthritis. Journal of Biomechanics 34, 907-915.

Kepple, T. M., Siegel, K. L., \& Stanhope, S. J., 1997. Relative contributions of the lower extremity joint moments to forward progression and support during gait. Gait \& Posture, 6, 1-8.

Krebs, D.E., Wong, D., Jevsevar, D., Riley, P.O., Hodge, W.A., 1992. Trunk kinematics during locomotor activities. Phys Ther, 72, 505-514.

Lin, Y.-C., Kim, H.J., Pandy, M.G., 2011. A computationally-efficient method for assessing muscle function during human locomotion. International Journal for Numerical Methods in Biomedical Engineering 27, 436-449.

Lin, Y.-C., Dorn, T.W., Schache, A.G., Pandy, M.G., 2012. Comparison of different methods for estimating muscle forces in human movement. Proceedings of the Institution of Mechanical Engineers, Part H: Journal of Engineering in Medicine, 226, 103-112.

Liu, M.Q., Anderson, F.C., Pandy, M.G., Delp, S.L., 2006. Muscles that support the body also modulate forward progression during walking. Journal of Biomechanics 39, 2623-2630.

Lu, T.W., O'Connor, J.J., 1999. Bone position estimation from skin marker coordinates using global optimisation with joint constraints. Journal of Biomechanics 32, 129-134.

Lyons, K., Perry, J., Gronley, J. K., Barnes, L., \& Antonelli, D., 1983. Timing and relative intensity of hip extensor and abductor muscle action during level and stair ambulation. An EMG study. Phys Ther 63, 1597-1605.

Manning, D.P., 1983. Deaths and injuries caused by slipping, tripping and falling. Ergonomics 26, 3-9.

McFadyen, B.J., Winter, D.A., 1988. An integrated biomechanical analysis of normal stair ascent and descent. Journal of Biomechanics, 21, 733-744.

Nadeau, S., McFadyen, B.J., Malouin, F., 2003. Frontal and sagittal plane analyses of the stair climbing task in healthy adults aged over 40 years: what are the challenges compared to level walking? Clinical Biomechanics 18, 950-959.

Neptune, R.R., Zajac, F.E., Kautz, S.A., 2004. Muscle force redistributes segmental power for body progression during walking. Gait and Posture 19, 194-205.

Perry, J., 1992. Gait analysis : normal and pathological function. Thorofare, N.J.: SLACK Incorporated.

Pandy, M. G., 2001. Computer modeling and simulation of human movement. Annu Rev Biomed Eng 3: 245-273.

Pandy, M.G., Andriacchi, T.P., 2010. Muscle and joint function in human locomotion. Annual Review of Biomedical Engineering 12, 401-433.

Pandy, M.G., Lin, Y.-C., Kim, H.J., 2010. Muscle coordination of mediolateral balance in normal walking. Journal of Biomechanics 43, 2055-2064.

Protopapadaki, A., Drechsler, W.I., Cramp, M.C., Coutts, F.J., Scott, O.M., 2007. Hip, knee, ankle kinematics and kinetics during stair ascent and descent in healthy young individuals. Clinical Biomechanics 22, 203-210.

Reeves, N.D., Spanjaard, M., Mohagheghi, A.A., Baltzopoulos, V., Maganaris, C.N., 2008. The demands of stair descent relative to maximum capacities in elderly 
and young adults. Journal of Electromyography and Kinesiology 18, 218227.

Riener, R., Rabuffetti, M., Frigo, C., 2002. Stair ascent and descent at different inclinations. Gait \& Posture 15, 32-44.

Seguin, R., Nelson, M.E., 2003. The benefits of strength training for older adults. American Journal of Preventive Medicine 25, 141-149.

Silverman, A. K., Neptune, R. R., Sinitski, E. H., \& Wilken, J. M., 2014. Whole-body angular momentum during stair ascent and descent. Gait Posture, 39, 11091114.

Svanström, L., 1974. Falls on stairs: an epidemiological accident study. Scandinavian Journal of Public Health 2, 113-120.

Tinetti, M.E., Speechley, M., Ginter, S.F., 1988. Risk-Factors for Falls among Elderly Persons Living in the Community. New England Journal of Medicine, 319, 1701-1707.

Winter, D.A., 1995. Human balance and posture control during standing and walking. Gait \& Posture 3, 193-214.

Whatling, G. M., \& Holt, C. A., 2010. Does the choice of stair gait cycle affect resulting knee joint kinematics and moments? Proceedings of the Institution of Mechanical Engineers Part H-Journal of Engineering in Medicine, 224(H9), 1085-1093.

Zachazewski, J. E., Riley, P. O., \& Krebs, D. E. (1993). Biomechanical analysis of body mass transfer during stair ascent and descent of healthy subjects. $\mathrm{J}$ Rehabil Res Dev, 30(4), 412-422.

Zajac, F.E., 1989. Muscle and tendon: properties, models, scaling, and application to biomechanics and motor control. Critical reviews in biomedical engineering 17, 359-411.

Zajac, F.E., Gordon, M.E., 1989. Determining muscle's force and action in multiarticular movement. Exercise and Sport Sciences Reviews, 17, 187-230. 
2 Fig. 1. Mean joint moments during level walking (black solid line), stair ascent (black

3 dashed line) and stair descent (black dotted line). Positive moments represent knee extension, hip flexion, hip abduction, hip internal rotation, ankle plantarflexion, and subtalar inversion.

Fig. 2. Comparison of experimental EMG and model-predicted muscle forces for level walking, stair ascent, and stair descent. The shaded areas represent the mean normalized EMG linear envelopes recorded for all participants, whereas the solid lines represent the mean muscle forces (dashed lines represent \pm one standard deviation from the mean) predicted for all participants. Symbols defining the major gait events are as follows: IHS, ipsilateral heel-strike; CTO, contralateral toe-off; CHS, contralateral heel-strike; and ITO, ipsilateral toe-off. Muscle symbols appearing in the graphs are: GMAX (superior, middle and inferior gluteus maximus), GMED (anterior, middle and posterior compartments of gluteus medius/minimus), VAS (vastus medialis, vastus intermedius and vastus lateralis), GAS (medial and lateral compartments of gastrocnemius), and SOL (soleus). For each muscle, the EMG linear envelopes are dimensionless and have been scaled using the same scaling factor for all three functional tasks to enhance clarity. The scaling factor for each muscle was determined by calculating the ratio between the peak muscle force and the peak normalized EMG activity across three functional tasks.

Fig. 3. Mean peak forces calculated for GMAX, VAS, SOL, GMED, and GAS during the first-half (top panel) and second-half (bottom panel) of stance in level walking and stair ambulation. The thin vertical line at the top of each histogram represents \pm 1 standard deviation from the mean. Asterisks and double asterisks indicate $p<0.017$ and $p<0.001$, respectively, for post hoc tests. 
1 Fig. 4. Comparison of contributions of major muscle groups to the vertical, fore-aft and mediolateral components of the center-of-mass acceleration for stair ambulation and level walking. The solid line (walking), dashed line (stair ascent) and dotted line (stair descent) represent the mean contributions of individual muscles to the center of mass acceleration in the vertical (top row), fore-aft (middle row) and mediolateral (bottom row) directions. Upward, forward and lateral accelerations are positive; downward, backward and medial accelerations are negative. OTHER represents the sum of the contributions from all lower-limb muscles included in the model other than GMAX, VAS, SOL, GMED, and GAS.

Fig. 5. Mean peak contributions of GMAX, VAS, SOL, GMED, and GAS to the vertical, fore-aft, and mediolateral accelerations of the COM during the first-half of stance in level walking and stair ambulation. Upward, forward and lateral accelerations are positive; downward, backward and medial accelerations are negative. The thin vertical line at the top of each histogram represents \pm 1 standard deviation from the mean. Asterisks and double asterisks indicate $p<0.017$ and $p<$ 0.001, respectively, for post hoc tests.

Fig. 6. Mean peak contributions of GMAX, VAS, SOL, GMED, and GAS to the vertical, fore-aft, and mediolateral accelerations of the COM during the second-half of stance in level walking and stair ambulation. Upward, forward and lateral accelerations are positive; downward, backward and medial accelerations are negative. The thin vertical line at the top of each histogram represents \pm 1 standard deviation from the mean. Asterisks and double asterisks indicate $p<0.017$ and $p<$ 0.001 , respectively, for post hoc tests.

Supplementary Fig. S1. Potential contributions of GMAX and SOL to the fore-aft COM acceleration for level walking, stair ascent, and stair descent. Forward and 
1 backward accelerations are positive and negative, respectively. The solid (walking), dashed (stair ascent) and dotted (stair descent) lines represent the means calculated for

3 all subjects.

4 Supplementary Fig. S2. Mean ground reaction forces measured for level walking,

5 stair ascent, and stair descent. The solid lines are the means calculated for all subjects,

6 while the shaded regions represent \pm 1 standard deviation from the mean.

7 Supplementary Fig. S3. Mean sagittal-plane joint kinematics measured for level

8 walking, stair ascent, and stair descent. Positive values represent hip flexion, knee

9 flexion, and ankle dorsiflexion. The solid lines are the means calculated for all

10 subjects, while the shaded regions represent \pm 1 standard deviation from the mean.

11 Supplementary Fig. S4. Mean frontal- and transverse-plane joint kinematics measured for level walking, stair ascent, and stair descent. Positive values represent hip adduction, hip internal rotation, and subtalar eversion. The solid lines are the means calculated for all subjects, while the shaded regions represent \pm 1 standard deviation from the mean. ascent, and stair descent. The solid lines are the means calculated for all subjects, while the shaded regions represent \pm 1 standard deviation from the mean. 

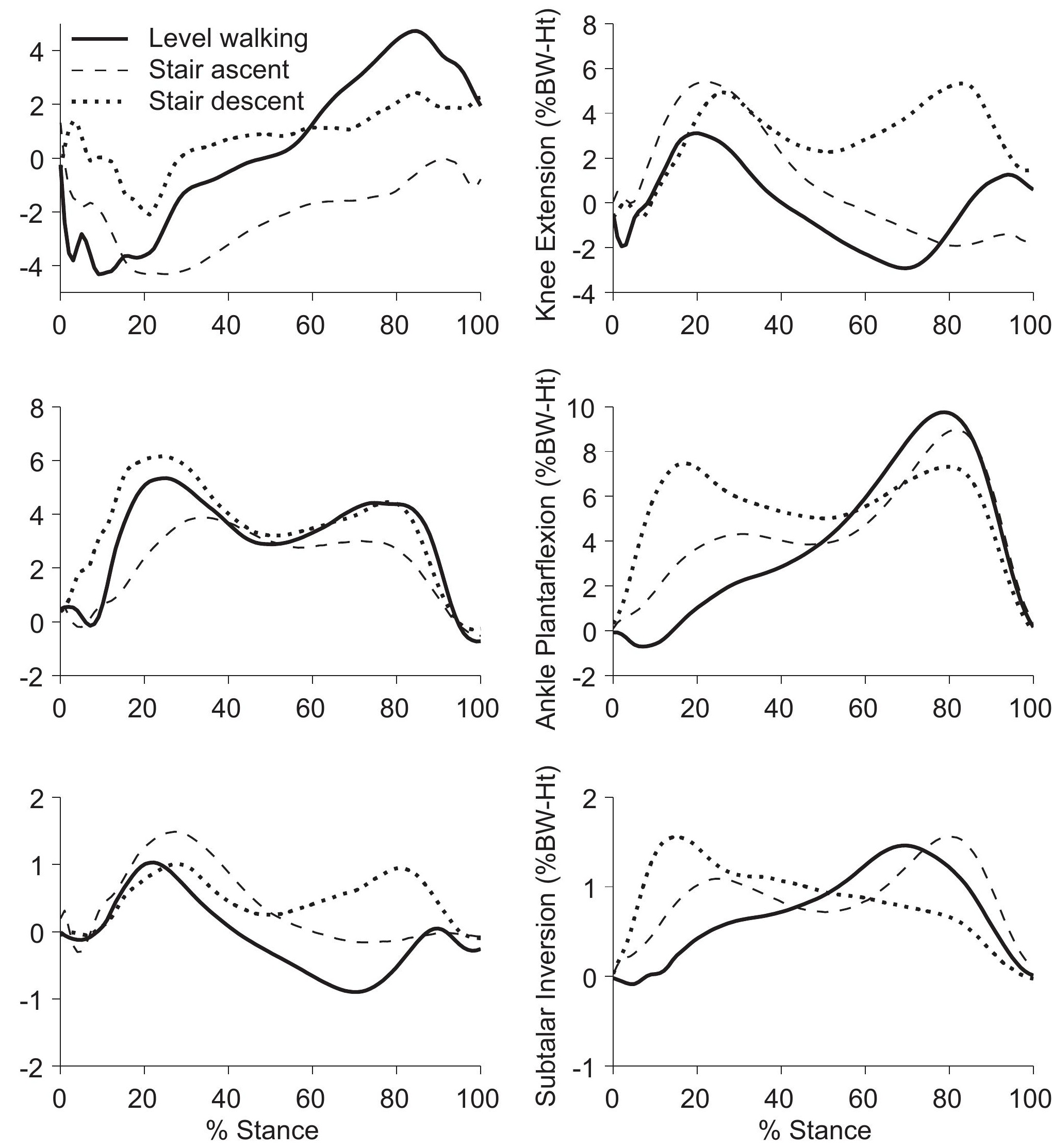
Level Walking

Stair Ascent

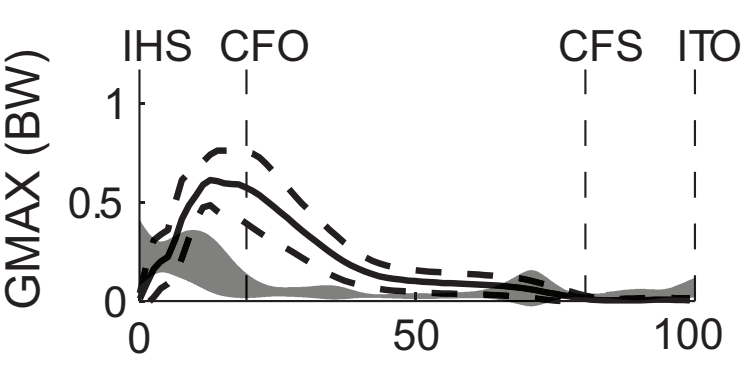

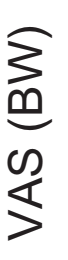

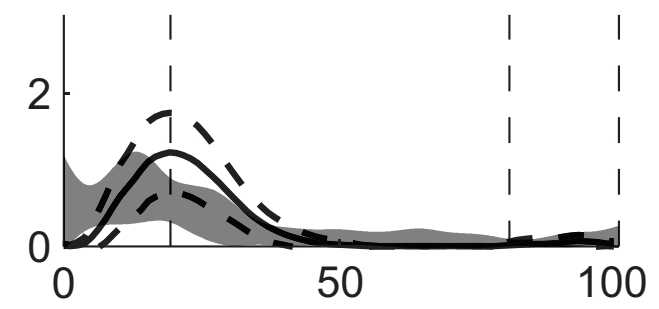

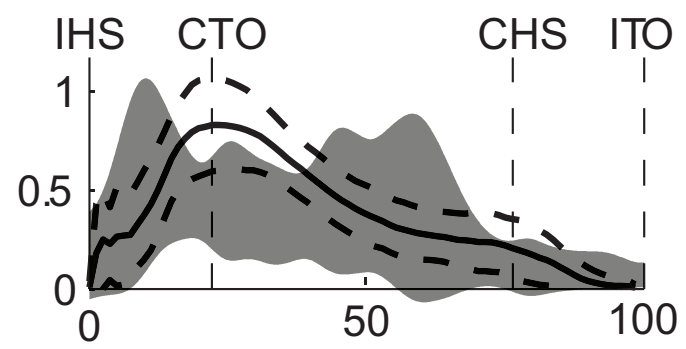

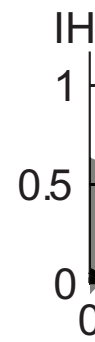

Stair Descent

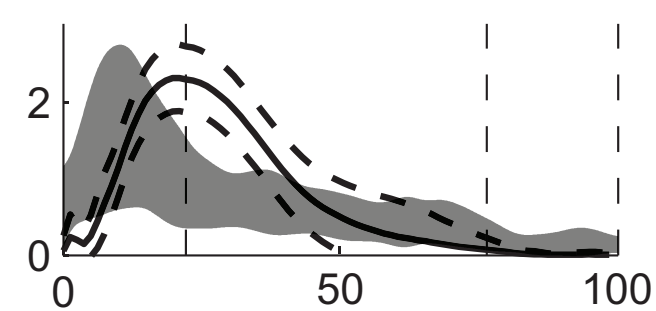

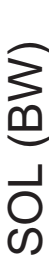
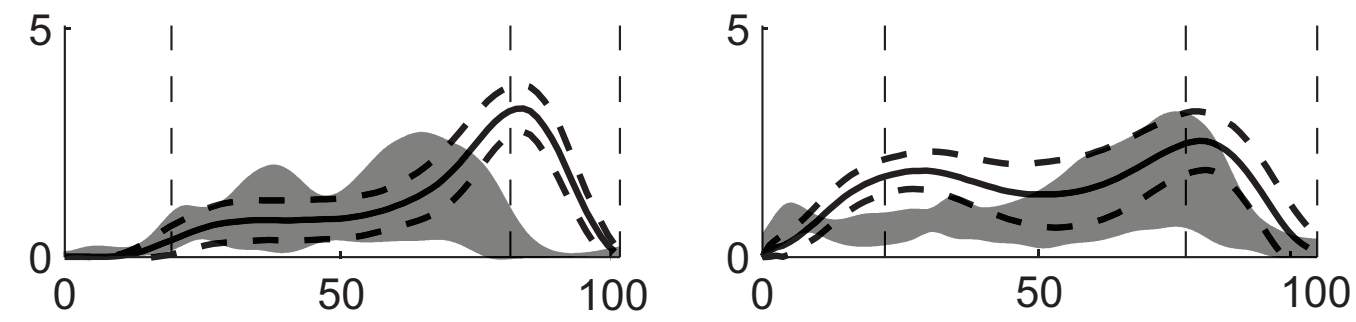

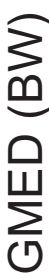
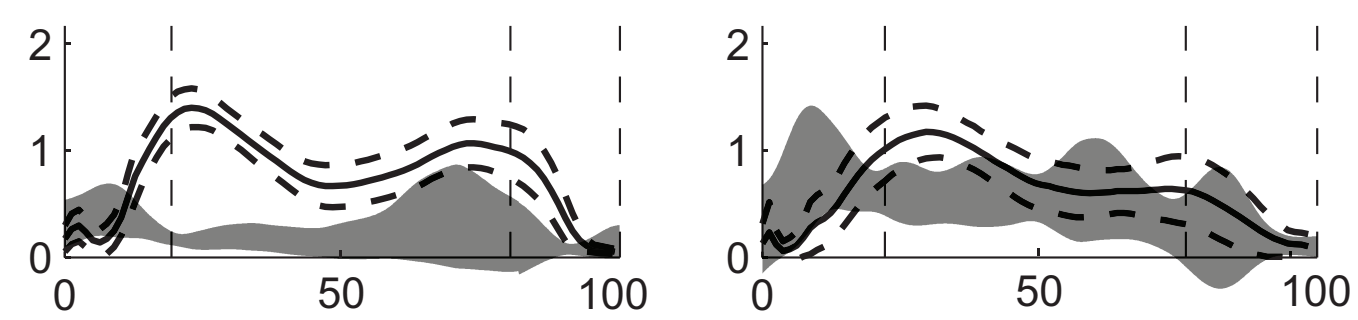

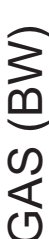

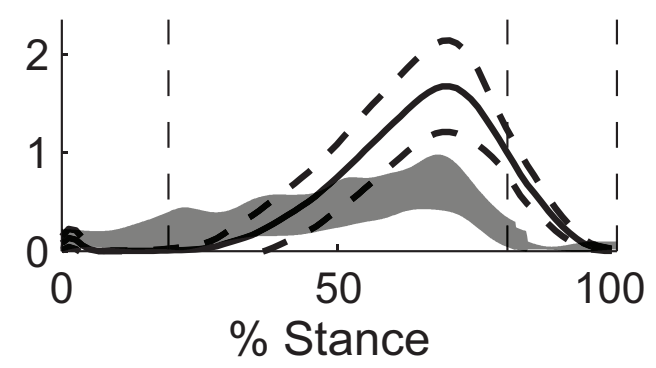

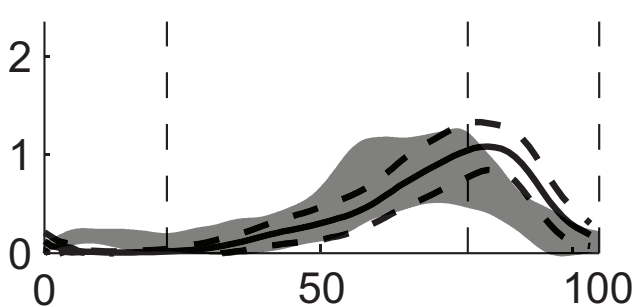

$\%$ Stance
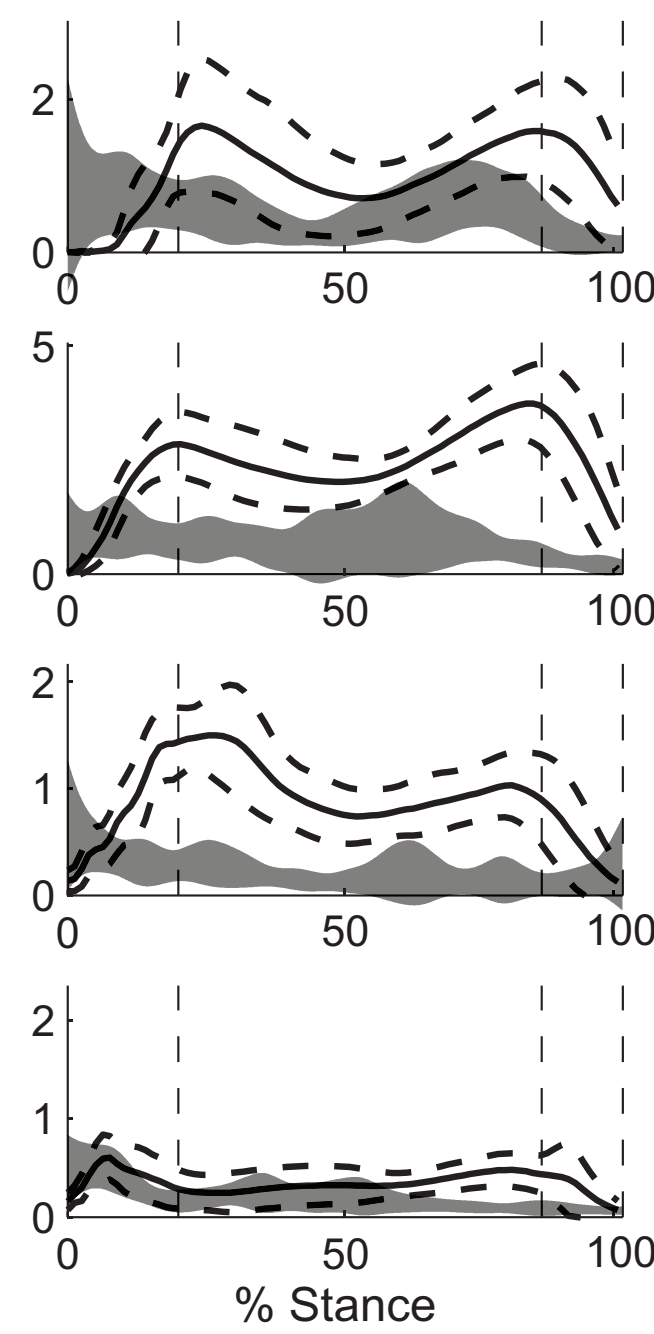


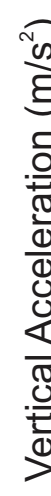

.

12 Level walking Stair ascent

Stair descent

**
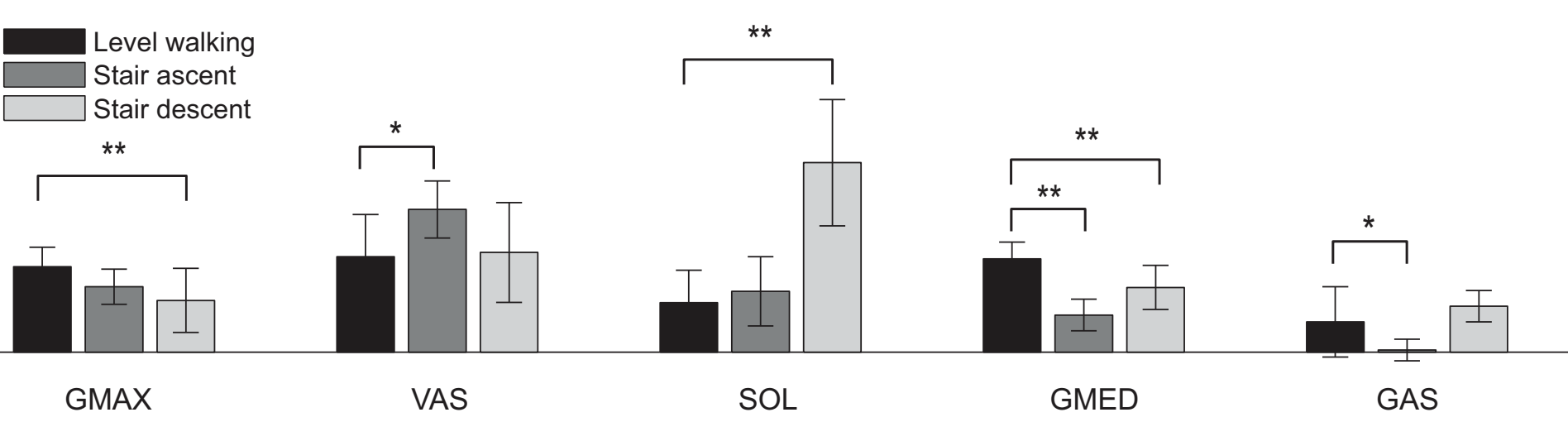

0

GMAX

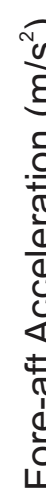
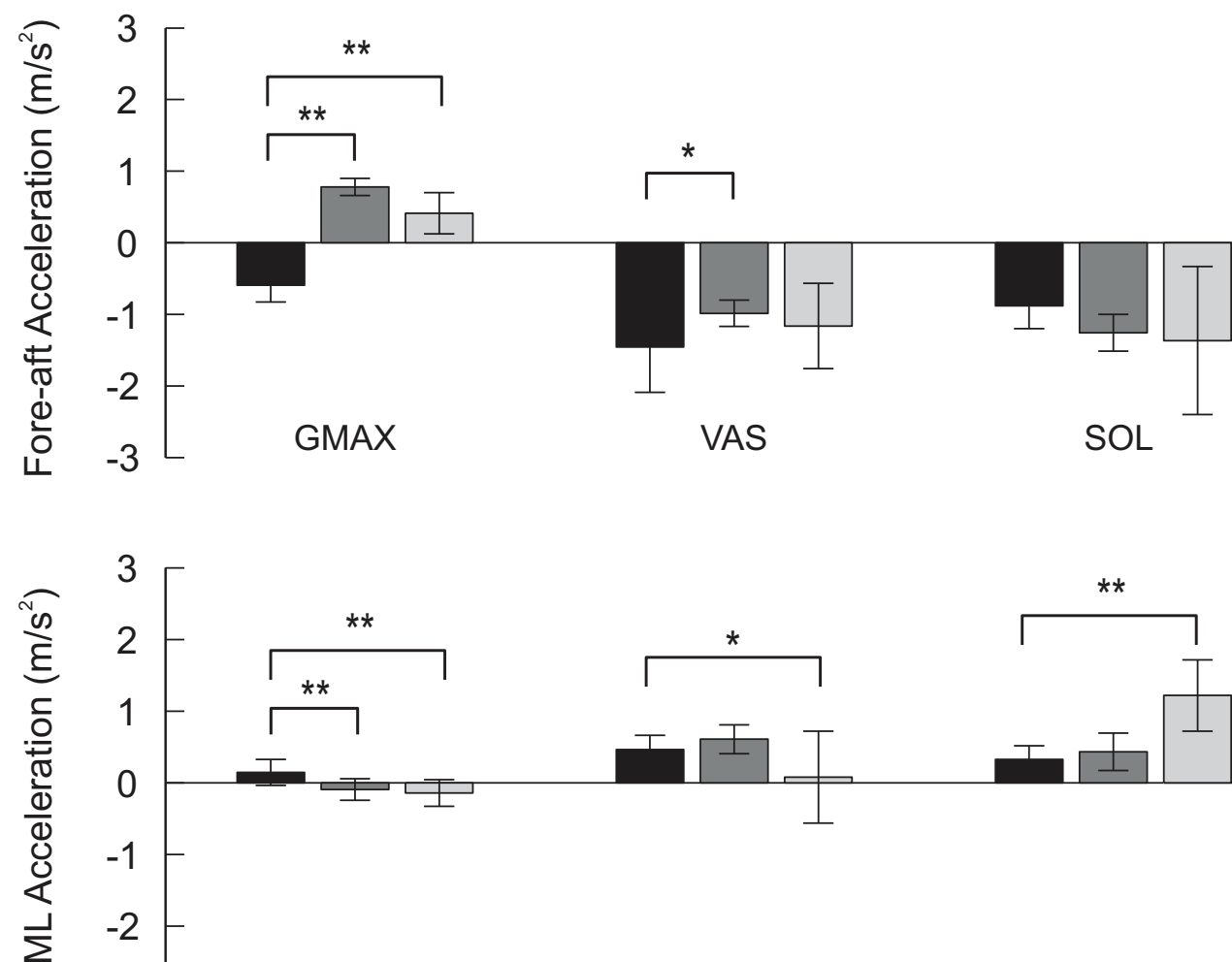

GMAX

VAS

GMED

GAS

**

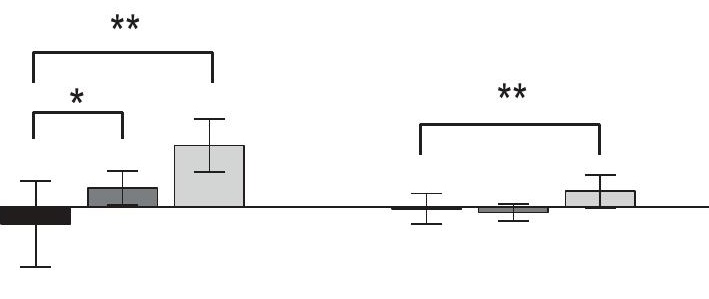

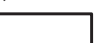

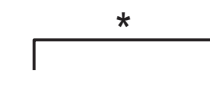

II

**

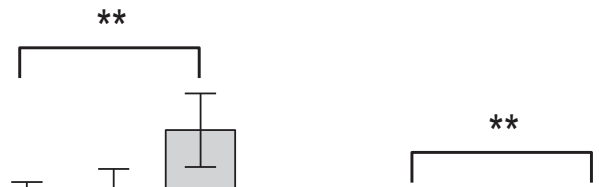

**
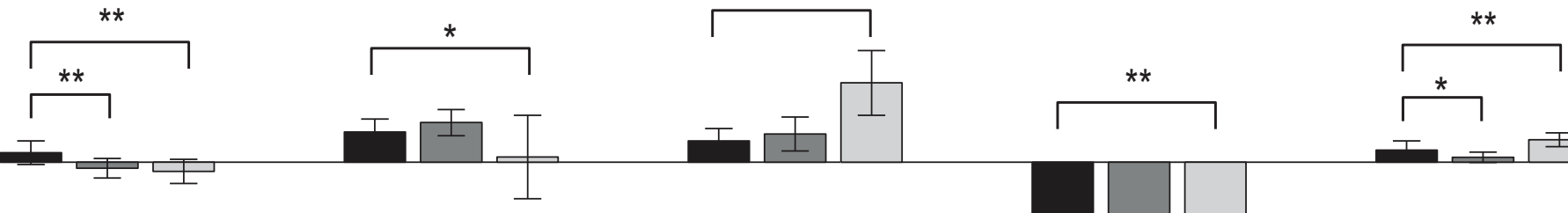

VAS

SOL 


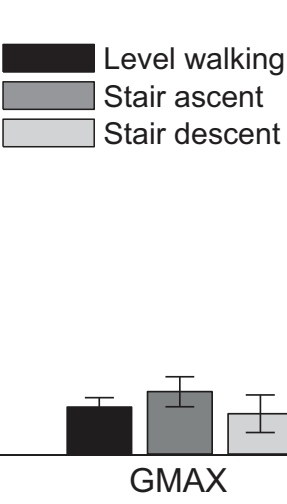

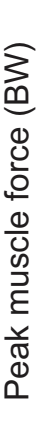
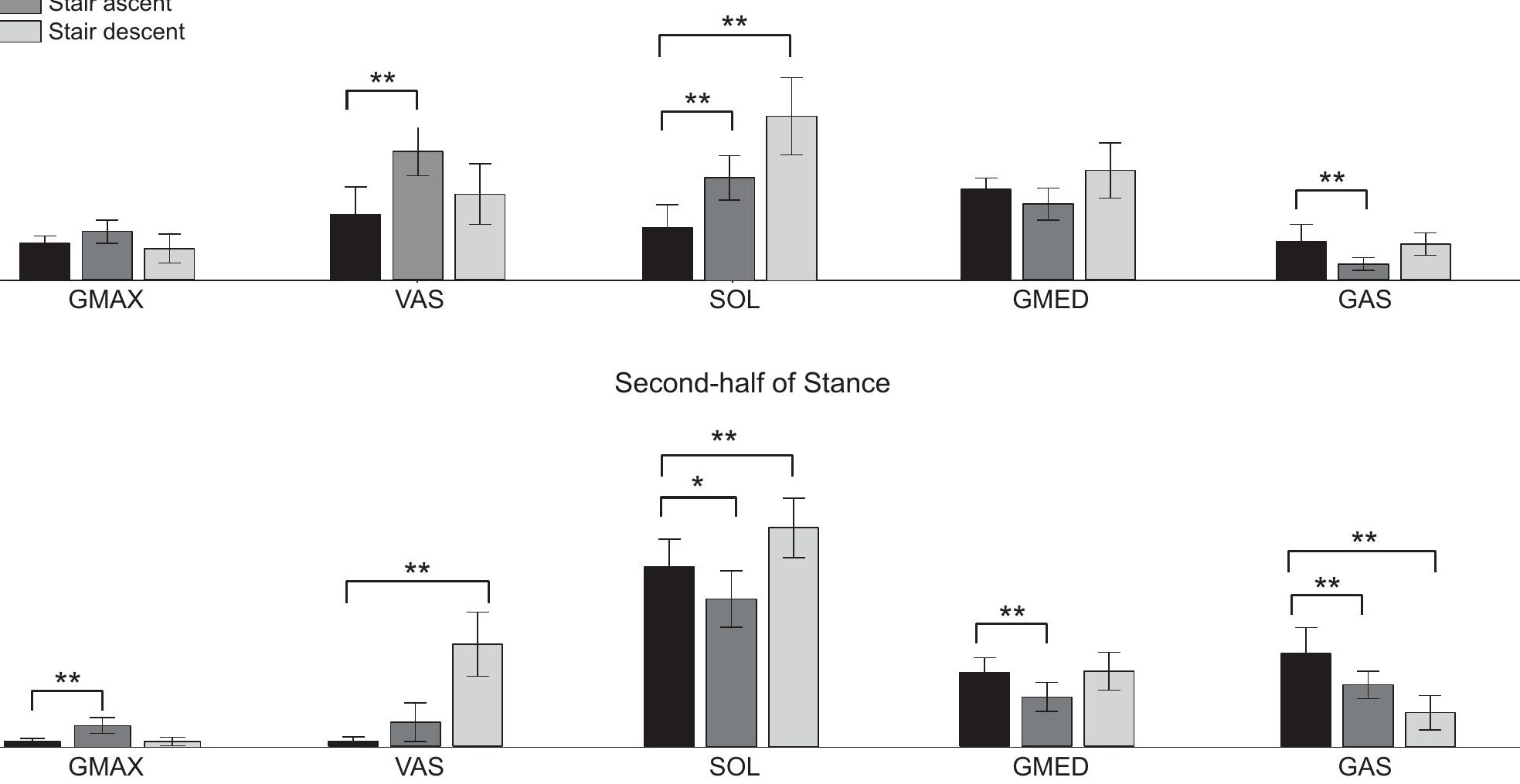

Second-half of Stance 


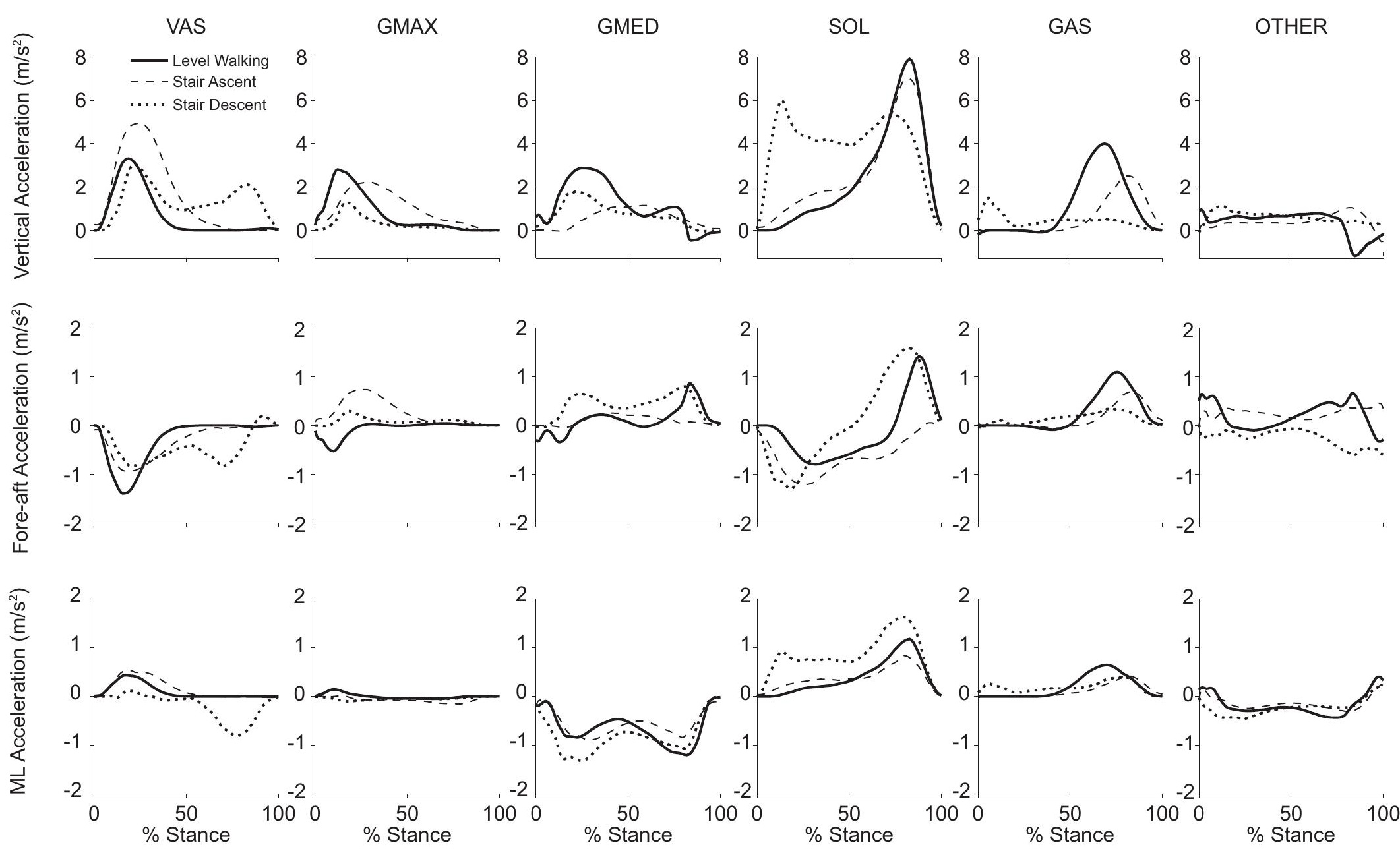


Table 1. A summary of p-values for the main effect of locomotor task on selected peak muscle forces and peak muscle contributions to the center-of-mass (COM) accelerations. All p-values were calculated using one-way repeated-measures ANOVA. Significance was set at $\mathrm{p}<0.017$ and indicated by the grey-shaded region.

\begin{tabular}{|c|c|c|c|c|c|c|c|c|}
\hline & \multicolumn{4}{|c|}{ First-half of stance } & \multicolumn{4}{|c|}{ Second-half of stance } \\
\hline & \multirow[t]{2}{*}{ Force } & \multicolumn{3}{|c|}{ COM acceleration } & \multirow[t]{2}{*}{ Force } & \multicolumn{3}{|c|}{ COM acceleration } \\
\hline & & Vertical & Fore-aft & Mediolateral & & Vertical & Fore-aft & Mediolateral \\
\hline$\overline{\text { GMAX }}$ & $<0.001$ & 0.002 & $<0.001$ & $<0.001$ & $<0.001$ & $<0.001$ & $<0.001$ & $<0.001$ \\
\hline VAS & $<0.001$ & 0.005 & 0.049 & 0.003 & $<0.001$ & $<0.001$ & $<0.001$ & $<0.001$ \\
\hline SOL & $<0.001$ & $<0.001$ & 0.107 & $<0.001$ & $<0.001$ & $<0.001$ & $<0.001$ & $<0.001$ \\
\hline GMED & $<0.001$ & $<0.001$ & $<0.001$ & $<0.001$ & $<0.001$ & 0.300 & $<0.001$ & 0.002 \\
\hline GAS & $<0.001$ & $<0.001$ & $<0.001$ & $<0.001$ & $<0.001$ & $<0.001$ & $<0.001$ & 0.004 \\
\hline
\end{tabular}




\section{University Library}

\section{- M M N E R VA A gateway to Melbourne's research publications}

Minerva Access is the Institutional Repository of The University of Melbourne

Author/s:

Lin, Y-C;Fok, LA;Schache, AG;Pandy, MG

Title:

Muscle coordination of support, progression and balance during stair ambulation

Date:

2015-01-21

Citation:

Lin, Y. -C., Fok, L. A., Schache, A. G. \& Pandy, M. G. (2015). Muscle coordination of support, progression and balance during stair ambulation. JOURNAL OF BIOMECHANICS, 48 (2), pp.340-347. https://doi.org/10.1016/j.jbiomech.2014.11.019.

Persistent Link:

http://hdl.handle.net/11343/55023 